\title{
Vibrational Relaxation Lifetime of a Physisorbed Molecule at a Metal Surface
}

\author{
Sumit Kumar, ${ }^{1, *}$ Hongyan Jiang, ${ }^{1, \dagger}$ Michael Schwarzer, ${ }^{4}$ Alexander Kandratsenka, ${ }^{1}$ \\ Dirk Schwarzer, ${ }^{1}$ and Alec M. Wodtke $\oplus^{1,2,3}$ \\ ${ }^{1}$ Department of Dynamics at Surfaces, Max-Planck-Institute for Biophysical Chemistry, Am Fassberg 11, 37077 Göttingen, Germany \\ ${ }^{2}$ Institute for Physical Chemistry, University of Göttingen, Tammannstrasse 6, 37077 Göttingen, Germany \\ ${ }^{3}$ International Center for Advanced Studies of Energy Conversion, Georg-August University of Göttingen, \\ Tammannstrasse 6, 37077 Göttingen, Germany \\ ${ }^{4}$ Institute of Aerodynamics and Flow technology, German Aerospace Center (DLR), \\ Bunsenstrasse 10, 37073 Göttingen, Germany
}

(Received 8 March 2019; published 10 October 2019)

\begin{abstract}
Previous measurements of vibrational relaxation lifetimes for molecules adsorbed at metal surfaces yielded values of 1-3 ps; however, only chemisorbed molecules have been studied. We report the first measurements of the vibrational relaxation lifetime of a molecule physisorbed to a metal surface. For $\mathrm{CO}(v=1)$ adsorbed on $\mathrm{Au}(111)$ at $35 \mathrm{~K}$ the vibrational lifetime of the excited stretching mode is $49 \pm 3$ ps. The long lifetime seen here is likely to be a general feature of physisorption, which involves weaker electronic coupling between the adsorbate and the solid due to bonding at larger distances.
\end{abstract}

DOI: 10.1103/PhysRevLett.123.156101

Introduction.-Vibrational excitation of gas-phase reactants can dramatically enhance reaction rates and control reaction pathways [1-3]. For chemical reactions on metal surfaces, vibrationally excited reactants are believed to thermalize with the solid much faster than dissociation, diffusion, and reaction. This is a central assumption of the Langmuir-Hinshelwood (LH) reaction mechanism [4-6], one of the foundations of heterogeneous catalysis. Nevertheless, some studies show that vibrationally excited molecules promote surface reactions even when the reaction is preceded by adsorption [7-9]. This raises the question: under what conditions is the thermalization assumption of the LH mechanism valid?

The rate of vibrational relaxation depends on the interactions of the molecule with the surface, which are thought to be well understood. When vibrationally excited $\mathrm{CO}$ is adsorbed on an insulator surface like $\mathrm{NaCl}$, millisecond lifetimes are observed [10]; the vibrating CO molecules behave like classical oscillating dipoles, losing their energy to the $\mathrm{NaCl}$ lattice through the electromagnetic near field [11]. When $\operatorname{CO}(v=1)$ is chemisorbed on semiconductors like silicon, the lifetime is a few nanoseconds, reflecting the anharmonic coupling of the $\mathrm{CO}$ stretch to the phonon bath through the chemical bond made to the surface [12]. The shortest lifetimes observed are those of molecules chemisorbed to metals: 2.2 and $2.0 \pm$ 1.0 ps for $\mathrm{CO}$ on $\mathrm{Pt}(111)$ [13] and $\mathrm{Cu}(100)$ [14], respectively. These short lifetimes result from Born-Oppenheimer breakdown, where vibrational energy is efficiently transferred to metal electronic excitation [15-28]. If this behavior was typical for adsorbates on metals, the thermalization assumption would be valid for $\mathrm{LH}$ reactions on a wide variety of important catalysts.
Physisorbed molecules interacting through dispersion forces with the metal are bound at longer distances and exhibit little or no electron exchange with the metal. Theory predicts physisorbed molecules exhibit longer vibrational relaxation lifetimes [29-33]. This has never been experimentally verified, as physisorbed molecules have very small spectroscopic transition strengths, making vibrational lifetime measurements difficult.

In this Letter, we report direct lifetime measurements for vibrationally excited molecules physisorbed to a metal surface. $\mathrm{CO}$ adsorbed on $\mathrm{Au}(111)$ exhibits an infrared $\mathrm{CO}$-stretch frequency quite close to that of gas-phase CO. This, as well as its low desorption temperature $(47 \mathrm{~K})$, is a characteristic of physisorption. We use sum frequency generation detection to monitor the population of $\mathrm{CO}(v=1$ and $v=0)$ after laser preparation of $\mathrm{CO}(v=1)$. Population in both states recovers with the same time constant, $49 \pm 3$ ps. This demonstrates that, when molecules interact with metal surfaces through dispersion forces, vibrational excitation can survive much longer than the typical times required for dissociation, diffusion, and reaction in surface chemistry. This suggests that physisorbed molecules may provide pathways for vibrationally promoted surface reactions, previously thought impossible.

Experimental.-We performed pump-probe experiments to derive the vibrational lifetime of $\mathrm{CO}(v=1)$ on a $\mathrm{Au}(111)$ surface at $35 \mathrm{~K}$. Resonant pulsed infrared excitation was followed by a pair of laser pulses - a tunable IR pulse and a $532 \mathrm{~nm}$ up-conversion pulse-generating its frequency sum. The sum frequency generation (SFG) was enhanced when the IR pulse was resonant with $\mathrm{CO}$ vibration. All pulses were $20 \mathrm{ps}$ in duration. 

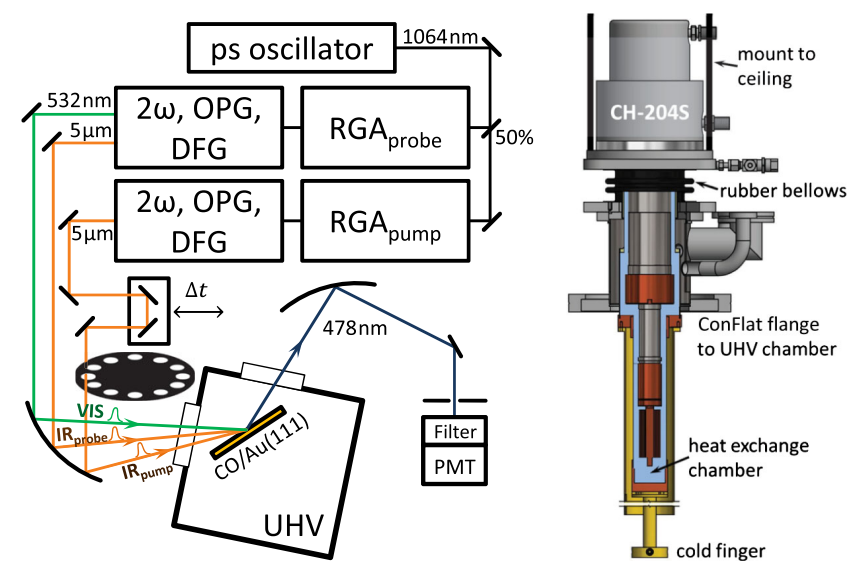

FIG. 1. Experimental setup to measure the vibrational lifetime of $\mathrm{CO}$ on $\mathrm{Au}(111)$ by surface pump-probe sum frequency generation. (Left) schematic diagram. (Right) Vibrational isolation for cryostatic cooling of the sample.

Figure 1 shows a schematic diagram of the experimental setup. Independently tunable IR pump and probe pulses were generated from two Nd:YAG-based $50 \mathrm{~Hz}$ regenerative amplifiers $\left(\mathrm{RGA}_{\text {pump }}\right.$ and $\left.\mathrm{RGA}_{\text {probe }}\right)$ seeded with a common Nd:YAG-based ps oscillator. The RGA outputs excited two optical parametric generators (OPGs) and difference frequency generators (DFGs), providing pulse energies up to $300 \mu \mathrm{J}$ at wavelengths close to $5 \mu \mathrm{m}$ and with a linewidth of $2 \mathrm{~cm}^{-1}$ (EKSPLA, PL2231-PG511DFG). Typically, 30 and $9 \mu \mathrm{J}$ pulses were used for $\mathrm{IR}_{\text {pump }}$ and $\mathrm{IR}_{\text {probe }}$, respectively, and $10 \mu \mathrm{J}$ pulses of $532 \mathrm{~nm}$ light were used in the SFG detection. All three laser beams were $p$ polarized and focused onto the $\mathrm{CO} / \mathrm{Au}(111)$ surface at an incidence angle of $\sim 80^{\circ}$ from the surface normal using a $90^{\circ}$-off-axis parabolic mirror ( $25 \mathrm{~cm}$ effective focal length). The SFG signal at $\sim 478 \mathrm{~nm}$ was collimated with a second parabolic mirror before it passed through an iris, a holographic notch filter centered at $532 \mathrm{~nm}$, and a short wave pass filter (Semrock, $532 \mathrm{~nm}$ RazorEdge). The remaining light was detected with a photomultiplier (PMT, Hamamatsu 1P28). The pump-probe time delay was varied with a computer controlled translation stage. The pump-induced change of the SFG intensity was measured by blocking every second $\mathrm{IR}_{\text {pump }}$ pulse with a synchronized chopper and averaging the signals with and without vibrational excitation of CO. Static SFG spectra were recorded as a function of the $\mathrm{IR}_{\text {probe }}$ frequency with the pump pulse blocked.

Infrared reflection absorption (IRRA) spectroscopy was also performed using an IR femtosecond laser system providing pulses with a $200 \mathrm{~cm}^{-1}$ bandwidth at $5 \mu \mathrm{m}$ (Coherent, Libra RGA, with home-built OPA and DFG). The laser beam was incident at the surface $86^{\circ}$ from the surface normal with a polarization rotated $45^{\circ}$ out of the incidence plane. The reflected light was resolved by a polarizer to give parallel $\left(I_{p}\right)$ and perpendicular $\left(I_{s}\right)$ intensities, whose spectra were independently measured with a spectrometer employing a liquid-nitrogen cooled $2 \times 32$ array $\mathrm{HgCdTe}$ detector (Infrared Associates, Inc.). Since only $p$-polarized radiation can be absorbed by the adsorbate, the $s$ component provides a pulse-to-pulse normalization reference to compensate laser intensity fluctuations. The reflectivity is calculated according to

$$
R(\nu)=\left(\frac{I_{p}(\nu)}{I_{s}(\nu)}\right) /\left(\frac{I_{p}(\nu)}{I_{s}(\nu)}\right)_{0},
$$

where the index 0 refers to a measurement from a clean $\mathrm{Au}(111)$ surface without $\mathrm{CO}$.

The Au single crystal [MaTeck, diameter $1.0 \mathrm{~cm}$, thickness $0.2 \mathrm{~cm}$, orientation accuracy of (111) surface $<0.1^{\circ}$, purity $99.999 \%$ ] was enclosed in an ultrahigh vacuum (UHV) chamber and mounted to a sample holder of similar design as that described in Ref. [34]. The UHV chamber is equipped with two $\mathrm{CaF}_{2}$ windows and pumped by a $550 \mathrm{l} / \mathrm{s}$ turbo pump (Leybold MAG W600iP), which is backed by a second $65 \mathrm{l} / \mathrm{s}$ turbo pump (Leybold Turbovac SL 80H) and an oil-based mechanical pump (Leybold Trivac D16B) providing a base pressure of $<1.5 \times 10^{-10}$ mbar. The $\mathrm{Au}$ crystal could be resistively heated (with a Heinzinger, PTN 32-20 power supply) or cooled to cryogenic temperatures using a closed cycle helium refrigerator (Janis research, CCS-XG-M/204N). The refrigerator was designed to reduce vibrations transferred from the cold head (SHI Sumitomo, model CH-204S) to the sample holder. The Joule-Thompson expander unit was mounted to the ceiling of the laboratory, while the sample mount was attached to the vacuum chamber that was mounted to the floor. A heat exchange chamber encapsulated by a rubber bellows filled with helium gas provided a thermal link between the expander and the sample mount. The Au crystal temperature was monitored with a type-E thermocouple inserted into a side hole of the crystal - the temperature was stabilized with a cryogenic temperature controller (Lakeshore, Model 336). The base temperature possible with this setup was $25 \mathrm{~K}$.

Prior to every SFG experiment, the $\mathrm{Au}(111)$ surface was cleaned for 25 min with a $3 \mathrm{kV} / 10 \mu \mathrm{A}$ argon ion sputtering beam (Specs $\mathrm{Ar}^{+}$gun, IQE 11/35). It was subsequently annealed at $1100 \mathrm{~K}$ for $30 \mathrm{~min}$. Sample cleanliness was verified using an Auger spectrometer (Staib Instruments, ESA 100). The CO monolayer sample was characterized by temperature programmed desorption (TPD). The monolayer desorbs at $47 \mathrm{~K}$ in agreement with previous work $[35,36]$. Overlayers desorb at $30 \mathrm{~K}$. For sample preparation and during the SFG experiment, the crystal was cooled to $35 \mathrm{~K}$ and dosed with a $\mathrm{CO}$ background pressure of $5 \times 10^{-8}$ mbar, admitted to the chamber by a leak valve. Under these conditions, a full CO monolayer is formed since molecules desorbed by the laser pulses are readily replaced, but $\mathrm{CO}$ multilayers are not formed. IRRA experiments were performed on $\mathrm{CO}$ monolayers at $25 \mathrm{~K}$ with the leak valve closed. Here, the measurement times were short 

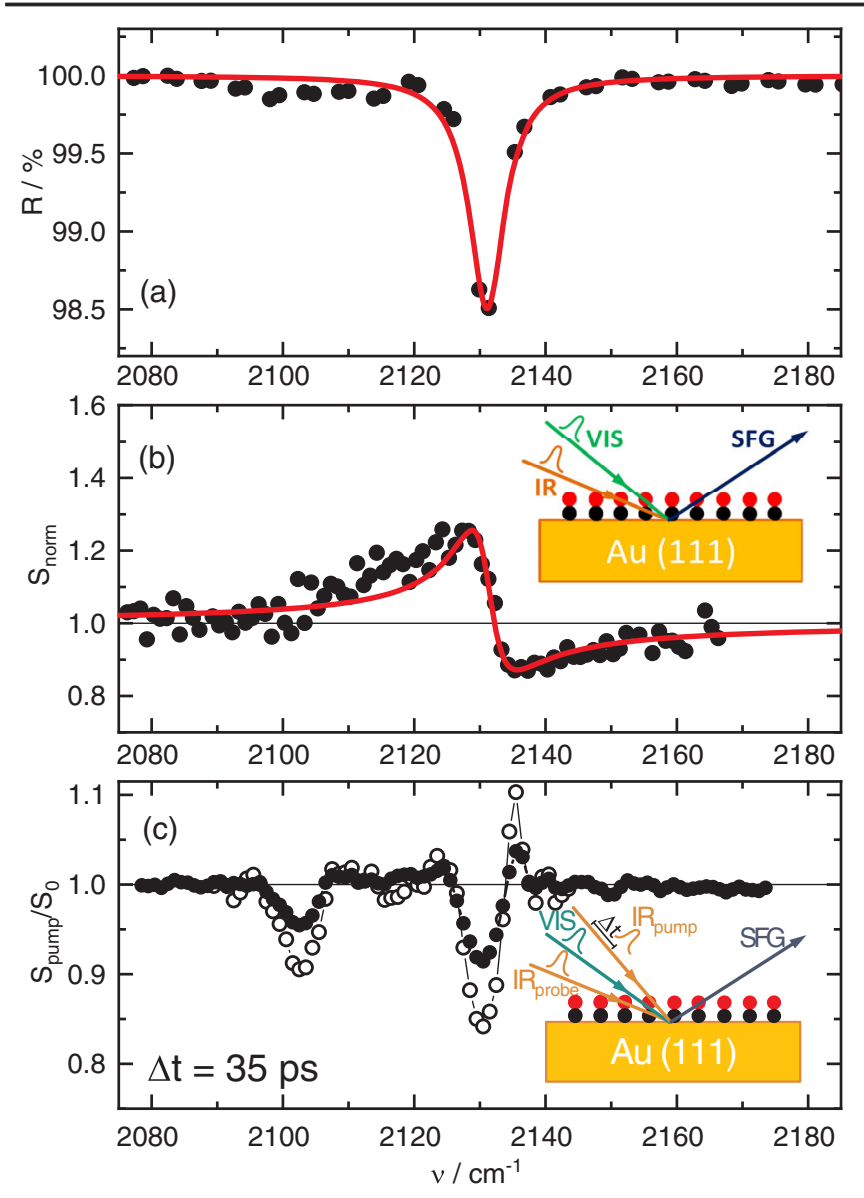

normalizing the spectrum obtained with a $\mathrm{CO}$ monolayer covering the surface $\left(\mathrm{SFG}_{\mathrm{ML}}\right)$ to a separately measured $\mathrm{SFG}$ spectrum from bare $\mathrm{Au}(111)\left(\mathrm{SFG}_{\mathrm{Au}}\right)$. The ratio $\mathrm{SFG}_{\text {norm }}=\mathrm{SFG}_{\mathrm{ML}} / \mathrm{SFG}_{\mathrm{Au}}$ was then calculated and modeled using Eq. (2) [40]

$I_{\mathrm{SFG}} \propto\left|\chi_{\mathrm{Au}}^{(2)}+\chi_{\mathrm{CO}}^{(2)}\right|^{2}=|| \chi_{\mathrm{Au}}^{(2)}\left|+\frac{a_{j}}{\left(\omega_{j}-\omega-i \Gamma_{j}\right)} e^{-i \varphi_{j}}\right|^{2}$,

where $\chi_{\mathrm{Au}}^{(2)}$ and $\chi_{\mathrm{CO}}^{(2)}$ are the $\mathrm{Au}$ and $\mathrm{CO}$ contribution to the surface's second-order susceptibility, respectively. The rhs of Eq. (2) is defined with $a_{j}, \omega_{j}$, and $\Gamma_{j}$ as oscillator strength, resonance frequency, and damping constant of the CO vibrational mode $j$, respectively. $\varphi_{j}$ is the phase difference between the $\mathrm{CO}$ and the Au contributions-the phase of the latter is assumed to be constant over the frequency range of our spectra and arbitrarily set to zero.

The data of Fig. 2 were fitted with a Lorentzian line shape function for the IRRA spectrum [red line, Fig. 2(a)], and Eq. (2) for the SFG spectrum [red line, Fig. 2(b)], using global parameters for $\mathrm{CO} v=0 \rightarrow 1$ resonance frequency and line width: $\nu_{01}=\omega_{01} /(2 \pi c)=2131 \mathrm{~cm}^{-1}$ and $\Delta \nu_{01}=$ $\Gamma_{01} /(\pi c)=6.5 \mathrm{~cm}^{-1}$. In addition, the fit to the SFG spectrum yields an amplitude ratio for $\mathrm{CO}$ to $\mathrm{Au}$ contribution: $a_{01} /\left|\chi_{\mathrm{Au}}^{(2)}\right|=0.19 \pm 0.02$ and the phase $\varphi_{01}=14^{\circ} \pm$ $4.0^{\circ}$. The SFG spectrum also shows enhanced intensity between 2100 and $2115 \mathrm{~cm}^{-1}$, indicating $\mathrm{CO}$ molecules adsorbed at defect sites of the surface [37].

Figure 2(c) shows changes in the SFG spectrum induced by a pump pulse resonant with the $\mathrm{CO} v=0 \rightarrow 1$ vibrational transition at $2131 \mathrm{~cm}^{-1}$ for a time delay between pump and probe $\Delta t=35 \mathrm{ps}$. While scanning the $\mathrm{IR}_{\text {probe }}$ wave number, the SFG intensity was recorded with and without the $\mathrm{IR}_{\text {pump }}$ pulse, yielding $S_{\text {pump }}$ and $S_{0}$, respectively. $S_{\text {pump }} / S_{0}$ is plotted in Fig. 2(c) and shows pumpinduced features at 2104, 2131, and $2135 \mathrm{~cm}^{-1}$. We assign the feature at $2131 \mathrm{~cm}^{-1}$ to the depletion of the $\mathrm{CO}$ ground state vibrational population and the $2104 \mathrm{~cm}^{-1}$ feature to the increase in $v=1$ population. The redshift of $27 \mathrm{~cm}^{-1}$ is consistent with the vibrational anharmonicity of $\mathrm{CO}$ [41]. We find that the ratio of intensities between the $0 \rightarrow 1$ and the $1 \rightarrow 2$ transitions is independent of pump pulse energy and that neither of the transitions is saturated. This linear dependence on population is a consequence of the fact that the SFG field from the CO sample is essentially heterodyne detected by interference with the much stronger SFG local oscillator field from the Au nonresonant background [42].

If the nonresonant background were much weaker than the resonant signal, the $\nu_{01}$ SFG intensity would be expected to be depleted after $0 \rightarrow 1$ pumping, whereas the $\nu_{12}$ signal would be enhanced [42]. Indeed this was seen for $\mathrm{H} / \mathrm{Si}(111)$ [43,44]. Interestingly, Fig. 2(c) shows that 

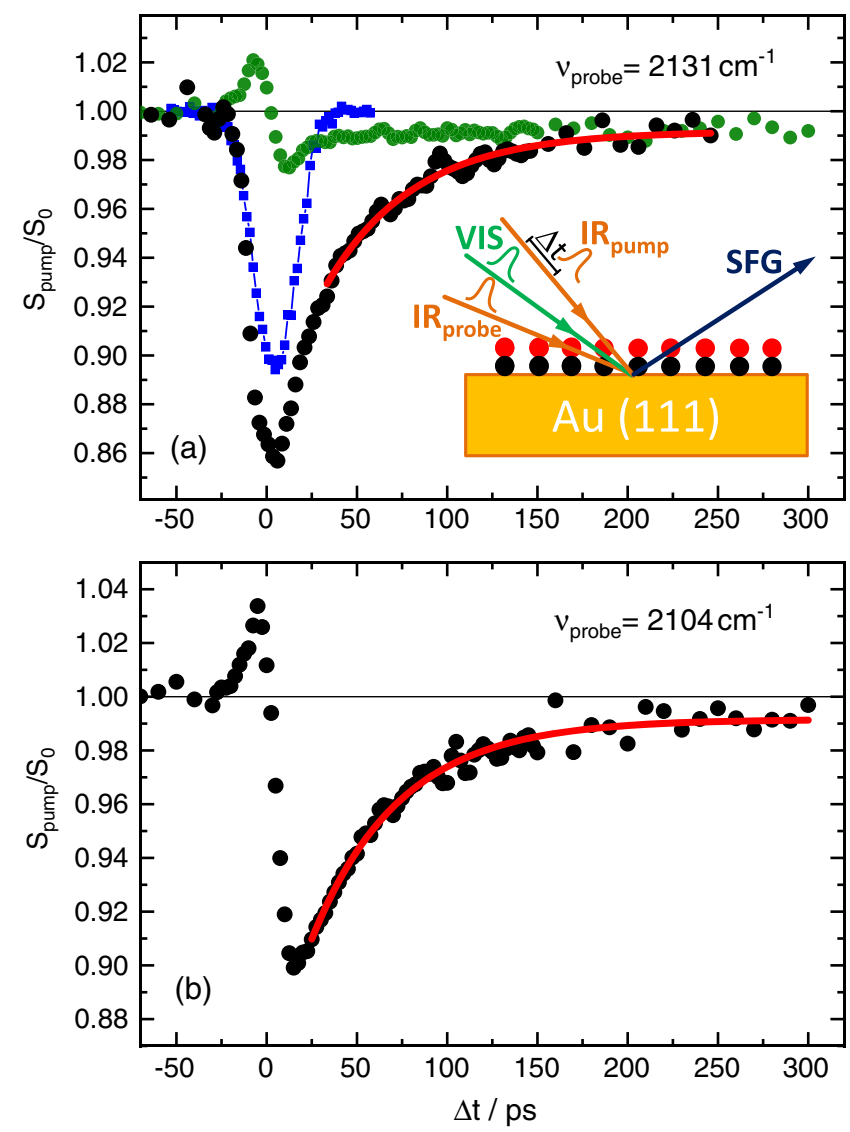

FIG. 3. Time-dependent SFG response of monolayer CO on $\mathrm{Au}(111)$ at $35 \mathrm{~K}$ with resonant excitation at $\nu_{\text {pump }}=2131 \mathrm{~cm}^{-1}$ and probing at (a) $\nu_{\text {probe }}=2131 \mathrm{~cm}^{-1}$ and (b) $2104 \mathrm{~cm}^{-1}$, respectively (black points). Red lines are obtained from least-square fits, yielding a vibrational lifetime of $\tau=49 \pm 3$ ps. (a) Green data points show the SFG response when the surface is excited off resonance from the $\mathrm{CO} 0 \rightarrow 1$ transition $\left(\nu_{\text {pump }}=2112 \mathrm{~cm}^{-1}\right)$. Blue squares represent the cross-correlation between the visible and IR pump pulse with a FWHM of 27 ps.

both signals move in the same direction. This can be rationalized if the phase for the $1 \rightarrow 2$ transition is $\varphi_{12} \sim$ $270^{\circ}$ (recall $\varphi_{01}=14^{\circ}$; see above). This phase difference may be induced by a modification of the Au-CO interaction potential upon $\mathrm{CO}$ vibrational excitation, leading to a change in the Au-CO equilibrium distance.

For a heterodyne detected SFG signal, the pump-pulseinduced intensity changes for the $0 \rightarrow 1$ and the $1 \rightarrow 2$ transitions are expected to have the same amplitude. In Fig. 2(c), however, the $0 \rightarrow 1$ feature is clearly enhanced compared to the $1 \rightarrow 2$ feature. In the Supplemental Material [45] we show that this results from a combination of two effects on the $0 \rightarrow 1$ transition, an $\mathrm{IR}_{\text {pump }}$-induced depletion of SFG intensity and a redshift of the excitonically coupled $0 \rightarrow 1$ band.

Figures 3(a) and 3(b) show the time dependence of these two signals after resonant excitation of the $\mathrm{CO}$ vibrational fundamental at $2131 \mathrm{~cm}^{-1}$ (black full circles). In both cases, the pump-induced SFG displays a single exponential decay with time constant $\tau_{1}=49 \pm 3$ ps (red curves). The green circles in Fig. 3(a) show the SFG response when the pump laser is tuned off resonance, $\nu_{\text {pump }}=2112 \mathrm{~cm}^{-1}$. We therefore assign the black data points of Figs. 3(a) and 3(b) to population relaxation from $v=1$ to $v=0$. To be precise, we measure the relaxation of exciton states of the adsorbed CO monolayer with dipole allowed transitions. As discussed in the Supplemental Material [45], these excitons arise from dipole-dipole coupling of the adsorbed $\mathrm{CO}$ molecules. The observation of a single exponential decay arising from a superposition of many dipole allowed transitions probing a manifold of exciton states indicates that these states are strongly coupled by dephasing with a characteristic time of $\sim 1$ ps (see Supplemental Material).

The lifetime observed in this Letter is much longer than seen in other related systems, where $\mathrm{CO}$ is chemisorbed to a metal. We next argue that the physisorption interaction between $\mathrm{CO}$ and $\mathrm{Au}(111)$ is responsible for the long lifetime.

Discussion.-There is strong evidence that CO is physisorbed on $\mathrm{Au}$. The desorption temperature of $\mathrm{CO}$ from $\mathrm{Au}(111)$ is $47 \mathrm{~K}[35,36]$ and the activation energy of desorption is $\sim 0.18 \mathrm{eV}$ [56]. At low temperature, this activation energy is a good measure of the binding energy - the derived small binding energy is consistent $\mathrm{CO}$ being physisorbed to $\mathrm{Au}(111)$. In backbonding of chemisorbed species, metal electron density moves to the $\mathrm{CO}$ antibonding $2 \pi^{*}$ orbital, simultaneously weakening the $\mathrm{C}-\mathrm{O}$ bond while strengthening the $\mathrm{C}-$ metal bond. We probe the strength of backbonding in our samples by infrared spectroscopy. This shows that backbonding is absent in $\mathrm{CO} / \mathrm{Au}$, as the $\mathrm{CO}$ vibrational frequency $\left(\nu_{01}=2131 \mathrm{~cm}^{-1}\right)$ is quite close to that of gas-phase CO $\left(2143 \mathrm{~cm}^{-1}\right)$, indicating little or no electron transfer to the $2 \pi^{*}$ orbital of CO. Strong backbonding leads to strongly redshifted $\nu_{01}$ values: for example, $2103 \mathrm{~cm}^{-1}$ [57] and $2070 \mathrm{~cm}^{-1}$ [58] for CO chemisorbed on Pt(111) and $\mathrm{Cu}(111)$, respectively. Further evidence that $\mathrm{CO}$ is physisorbed to $\mathrm{Au}$ comes from density functional theory calculations that predict a binding energy of $0.1 \mathrm{eV}$ [33], similar to the experimentally derived activation energy of desorption. Thus, all available evidence supports the conclusion that $\mathrm{CO}$ binds to $\mathrm{Au}(111)$ by dispersion forces.

There exists a strong theoretical foundation for the idea that a physisorbed molecule could have a much longer vibrational lifetime than its chemisorbed analogs. First, known mechanisms of vibrational relaxation are likely to shut down at the long binding distances typical of physisorbed molecules. For example, highly efficient vibrational relaxation can occur through electron transfer from the metal to the molecule forming a transient negative ion [15-21], a process which is strongly distance dependent due to image charge interaction. This is clearly described in Fig. 4 of Ref. [15] and Fig. 2 of Ref. [16]. In essence, the longer range repulsion associated with physisorption limits 
image charge stabilization of the negative ion state, thus preventing electron transfer. When electron transfer is impossible, we may model the interactions between an oscillating dipole and a jellium electron gas [29-32] or by density functional perturbation theory following Fermi's golden rule expression for the nonadiabatic vibrational damping [33]. These methods predict vibrational relaxation lifetimes of $\mathrm{CO}$ on $\mathrm{Au}(111)$ between 250 ps [33] and 0.5-2 ns [29-32] (see Supplemental Material [45]). These theories support the idea that physisorbed molecules' vibrational lifetimes are long; however, they are not in agreement with the $49 \pm 3$ ps lifetime observed in this Letter at $35 \mathrm{~K}$. Further theoretical work is required.

Thus, we conclude that physisorbed $\mathrm{CO}(v=1)$ may, by relaxing, still excite the metal's electron-hole pairs, but, due to its large distance to the metal, the strongest interactions are not possible. We emphasize that this explanation suggests there will be similar behavior for other physisorbed molecules.

Our measurements also shed light on recently reported molecular beam experiments [56], where $\mathrm{CO}(v=1$ and 2) was observed to desorb back to the gas phase after $\mathrm{CO}(v=2)$ adsorbed to $\mathrm{Au}(111)$. The degree of relaxation could not be quantified as the fraction of the $\mathrm{CO}$ sample desorbing, as $\mathrm{CO}(v=0)$ could not be measured. By assuming that the vibrational lifetime was comparable to thermal desorption lifetimes (100 ps), the order of magnitude of the vibrational lifetime could be estimated. This estimate suffered additional large uncertainties associated with the fact that the desorption rates used in the analysis were extrapolated from TPD measurements performed at much lower temperatures. The ambiguities and limitations inherent to this analysis are overcome in the present Letter, providing a quantitative measurement of the vibrational lifetime.

It is worth mentioning why similar results have not been obtained previously. Consider the magnitude of the infrared transition dipole moments for the internal stretching mode of a chemisorbed $\mathrm{CO}$ molecule that transfers electron density between the metal surface and the $\mathrm{CO}$ adsorbate during the course of $\mathrm{CO}$ vibration due to a backbonding interaction. This transition dipole moment is clearly much larger than that of an isolated $\mathrm{CO}$ gas-phase molecule, which a physisorbed $\mathrm{CO}$ more closely resembles. We carried out comparative measurements showing the peak infrared absorption cross section of $\mathrm{CO}$ on $\mathrm{Au}(111)$ is $\sim 0.1$ that of $\mathrm{CO}$ on $\mathrm{Pt}(111)$. In day-to-day operation, CO-SFG signals were far easier to find on $\mathrm{Pt}$ than on $\mathrm{Au}$. It is not surprising then that no vibrational lifetimes of molecules physisorbed to a metal have been previously reported, since experiments with such species are inherently more difficult, in particular, for physisorbed $\mathrm{CO}$ with its low desorption temperature. More sensitive detection methods allowing additional measurements on systems like this would be of great value in establishing the generality of our findings.
We thank Dr. Tim Schäfer who originally suggested making these measurements. We also acknowledge Jascha Lau for helpful discussions.

*Present address: Department of Chemistry, Anugrah Memorial College, Gaya, Magadh University, Bodhgaya 824234, India.

${ }^{\dagger}$ To whom all correspondence should be addressed. hongyan.jiang@mpibpc.mpg.de

[1] F. F. Crim, Science 249, 1387 (1990).

[2] F. F. Crim, J. Phys. Chem. 100, 12725 (1996).

[3] A. Sinha, M. C. Hsiao, and F. F. Crim, J. Chem. Phys. 94, 4928 (1991).

[4] I. Langmuir, J. Am. Chem. Soc. 35, 105 (1913).

[5] I. Langmuir, J. Am. Chem. Soc. 37, 1139 (1915).

[6] C. N. Hinshelwood and C. R. Prichard, J. Chem. Soc. 127, 327 (1925).

[7] A. V. Walker and D. A. King, J. Chem. Phys. 112, 4739 (2000).

[8] D. C. Seets, M. C. Wheeler, and C. B. Mullins, Chem. Phys. Lett. 266, 431 (1997).

[9] E. Dombrowski, E. Peterson, D. Del Sesto, and A. L. Utz, Catal. Today 244, 10 (2015).

[10] H.-C. Chang and G. E. Ewing, Phys. Rev. Lett. 65, 2125 (1990).

[11] L. Chen, J. A. Lau, D. Schwarzer, J. Meyer, V. B. Verma, and A. M. Wodtke, Science 363, 158 (2019).

[12] K. Laß, X. Han, and E. Hasselbrink, J. Chem. Phys. 123, 051102 (2005).

[13] J. Beckerle, R. R. Cavanagh, M. Casassa, E. J. Heilweil, and J. C. Stephenson, J. Chem. Phys. 95, 5403 (1991).

[14] M. Morin, N. J. Levinos, and A. L. Harris, J. Chem. Phys. 96, 3950 (1992).

[15] R. J. V. Wagner, N. Henning, B. C. Kruger, G. B. Park, J. Altschaffel, A. Kandratsenka, A. M. Wodtke, and T. Schafer, J. Phys. Chem. Lett. 8, 4887 (2017).

[16] Y. H. Huang, C. T. Rettner, D. J. Auerbach, and A. M. Wodtke, Science 290, 111 (2000).

[17] N. Shenvi, S. Roy, and J. C. Tully, Science 326, 829 (2009).

[18] N. Bartels, K. Golibrzuch, C. Bartels, L. Chen, D. J. Auerbach, A. M. Wodtke, and T. Schafer, Proc. Natl. Acad. Sci. U.S.A. 110, 17738 (2013).

[19] N. Bartels, B. C. Kruger, D. J. Auerbach, A. M. Wodtke, and T. Schafer, Angew. Chem., Int. Ed. Engl. 53, 13690 (2014).

[20] J. W. Gadzuk, J. Chem. Phys. 79, 6341 (1983).

[21] A. M. Wodtke, D. Matsiev, and D. J. Auerbach, Prog. Surf. Sci. 83, 167 (2008).

[22] M. Head-Gordon and J. C. Tully, J. Chem. Phys. 103, 10137 (1995).

[23] M. Head-Gordon and J. C. Tully, J. Chem. Phys. 96, 3939 (1992).

[24] V. Krishna and J. C. Tully, J. Chem. Phys. 125, 054706 (2006).

[25] J. W. Gadzuk and J. K. Norskov, J. Chem. Phys. 81, 2828 (1984).

[26] K. Golibrzuch, N. Bartels, D. J. Auerbach, and A. M. Wodtke, Annu. Rev. Phys. Chem. 66, 399 (2015).

[27] A. M. Wodtke, Chem. Soc. Rev. 45, 3641 (2016). 
[28] E. Hasselbrink, Curr. Opin. Solid State Mater. Sci. 10, 192 (2006).

[29] B. Persson, J. Phys. C 11, 4251 (1978).

[30] B. Persson and M. Persson, Surf. Sci. 97, 609 (1980).

[31] B. N. J. Persson and S. Andersson, Phys. Rev. B 29, 4382 (1984).

[32] A. Liebsch, Phys. Rev. Lett. 54, 67 (1985).

[33] I. Loncaric, M. Alducin, J. I. Juaristi, and D. Novko, J. Phys. Chem. Lett. 10, 1043 (2019).

[34] Q. Ran, D. Matsiev, A. M. Wodtke, and D. J. Auerbach, Rev. Sci. Instrum. 78, 104104 (2007).

[35] J. M. Gottfried, K. J. Schmidt, S. L. M. Schroeder, and K. Christmann, Surf. Sci. 536, 206 (2003).

[36] D. P. Engelhart, R. J. V. Wagner, A. Meling, A. M. Wodtke, and T. Schäfer, Surf. Sci. 650, 11 (2016).

[37] J. Pischel and A. Pucci, J. Phys. Chem. C 119, 18340 (2015).

[38] C. Ruggiero and P. Hollins, J. Chem. Soc., Faraday Trans. 92, 4829 (1996).

[39] C. Ruggiero and P. Hollins, Surf. Sci. 377-379, 583 (1997).

[40] Y. R. Shen, Proc. Natl. Acad. Sci. U.S.A. 93, 12104 (1996).

[41] A. W. Mantz and J. P. Maillard, J. Mol. Spectrosc. 57, 155 (1975).

[42] E. H. G. Backus, J. D. Cyran, M. Grechko, Y. Nagata, and M. Bonn, J. Phys. Chem. A 122, 2401 (2018).

[43] C. Hess, M. Wolf, and M. Bonn, Phys. Rev. Lett. 85, 4341 (2000).

[44] P. Guyot-Sionnest, Phys. Rev. Lett. 67, 2323 (1991).

[45] See Supplemental Material at http://link.aps.org/ supplemental/10.1103/PhysRevLett.123.156101 for (1) A model for excitonically coupled $\mathrm{CO}$ molecules in the monolayer. (2) An estimation of the vibrational lifetime based on theories by Persson and others, which includes Refs. [46-55].

[46] R. Disselkamp, H.-C. Chang, and G. E. Ewing, Surf. Sci. 240, 193 (1990).

[47] D. J. Dai and G. E. Ewing, Surf. Sci. 312, 239 (1994).

[48] S. Woutersen and P. Hamm, J. Phys. Condens. Matter 14, R1035 (2002).

[49] A. S. Davydov, Theory of Molecular Excitations (Plenum, New York, 1971).

[50] P. Hamm, M. Lim, and R. M. Hochstrasser, J. Phys. Chem. B 102, 6123 (1998).

[51] H.-J. Werner, Mol. Phys. 44, 111 (1981).

[52] H.-J. Chen, J. Wu, H. Liu, and X.-L. Cheng, Chin. Phys. B 24, 083102 (2015).

[53] M. Gajdoš, A. Eichler, and J. Hafner, J. Phys. Condens. Matter 16, 1141 (2004).

[54] C. Kittel, Introduction to Solid State Physics, 8th ed. (Wiley, New York, 2005).

[55] R. J. Maurer, M. Askerka, V. S. Batista, and J. C. Tully, Phys. Rev. B 94, 115432 (2016).

[56] P. R. Shirhatti, I. Rahinov, K. Golibrzuch, J. Werdecker, J. Geweke, J. Altschäffel, S. Kumar, D. J. Auerbach, C. Bartels, and A. M. Wodtke, Nat. Chem. 10, 592 (2018).

[57] J. V. Nekrylova, C. French, A. N. Artsyukhovich, V. A. Ukraintsev, and I. Harrison, Surf. Sci. Lett. 295, L987 (1993).

[58] B. E. Hayden, K. Kretzschmar, and A. M. Bradshaw, Surf. Sci. 155, 553 (1985). 